\title{
Episodic Real-Time CGM Use in Adults with Type 2 Diabetes: Results of a Pilot Randomized Controlled Trial
}

\author{
David A. Price (1) - Qianqian Deng · Mark Kipnes · Stayce E. Beck
}

Received: April 19, 2021 / Accepted: May 20, 2021 / Published online: June 4, 2021

(C) The Author(s) 2021

\begin{abstract}
Introduction: Resistance to initiating insulin therapy is common for people with type 2 diabetes (T2D) using multiple oral agents, resulting in sustained poor glycemic control. We explored a non-pharmacologic option and examined whether adults with T2D and elevated hemoglobin $\mathrm{A} 1 \mathrm{c}(\mathrm{HbA1c})$ who were using multiple, non-insulin antihyperglycemics could obtain glycemic benefit from limited, episodic use of real-time continuous glucose monitoring (rtCGM).

Methods: A randomized, pilot trial enrolled patients with T2D who were using two or more non-insulin therapies and had HbA1c values of 7.8-10.5\%. Following a baseline, 10-day, blinded CGM session, participants were randomized 2:1, rtCGM or self-monitoring of blood glucose (SMBG). Medication changes were not made during the 12-week study unless required for safety; benefits would result from lifestyle
\end{abstract}

Supplementary Information The online version contains supplementary material available at https:// doi.org/10.1007/s13300-021-01086-y.

D. A. Price $(\varangle) \cdot$ Q. Deng · S. E. Beck

Dexcom, Inc., 6340 Sequence Dr., San Diego, CA 92121, USA

e-mail: dprice@dexcom.com

M. Kipnes

Diabetes \& Glandular Disease Clinic,

5107 Medical Dr, San Antonio, TX 78229, USA changes. The rtCGM group used unblinded rtCGM for three sessions at weeks 0,4 , and 8 , and the control group managed diabetes with SMBG and wore blinded rtCGM at week 8 . Glycemic endpoints were assessed.

Results: Seventy participants were enrolled from eight North American sites and data were available from 68 ( $n=45 \mathrm{rtCGM} ; n=23 \mathrm{SMBG})$. Median (IQR) baseline HbA1c was $8.4(0.8) \%$ and $8.3(1.2) \%$ and median (IQR) change in HbA1c at week 12 was $-0.5(1.3) \%$ and -0.2 $(1.1) \%$ for the rtCGM and SMBG groups, respectively (between-group difference $p=0.74)$. More than one-third $(34.1 \%)$ of the rtCGM group vs $17.4 \%$ of the SMBG group reached the HbA1c goal of less than $7.5 \%$ at week 12 (between-group difference $p=0.12$ ). Compared to run-in, mean (SD) time in range (TIR 70-180 mg/dL) at week 8 increased for the rtCGM group (56.3 [24.5]\% vs 63.1 [25.5]\%) while it decreased for the SMBG group (68.4 [21.5]\% vs 55.1 [30.3]\%). HbA1c reductions were not sustained at month 9 .

Conclusion: In this pilot study, limited episodic rtCGM use in people failing multiple non-insulin therapies resulted in modest, short-term glycemic benefits.

Keywords: Episodic continuous glucose monitoring; HbA1c; Oral antihyperglycemic medications; T2D 


\section{Key Summary Points}

Why carry out this study

Suboptimal glycemic control is common among adults with type 2 diabetes (T2D) and therapy adjustments are often required to optimize control.

Episodic use of real-time continuous glucose monitoring (rtCGM) has been shown to significantly improve HbA1c in patients with T2D not on prandial insulin but CGM use patterns in prior studies do not match current reimbursement models or established CPT codes.

The COMMITED study was a pilot, randomized trial designed to be translatable to routine medical practice and to determine if episodic use of rtCGM in patients failing multiple non-insulin medications would facilitate improved lifestyle choices and/or better medication adherence and result in glycemic improvement compared to selfmonitoring of blood glucose (SMBG).

\section{What was learned from the study?}

Median baseline $\mathrm{HbA1c}$ was $8.4 \%$ and $8.3 \%$ and median change in HbA1c at week 12 was $-0.5 \%$ and $-0.2 \%$ for the rtCGM and SMBG groups, respectively (between-group difference $p=0.74$ ).

More than a third (34.1\%) of participants in the rtCGM group and $17.4 \%$ in the control group reached an HbA1c of less than $7.5 \%$ at week 12 . In other words, episodic rtCGM use (one 10-day wear per month for 3 months) enabled more than one-third of participants, who had poor glycemic control despite use of multiple oral agents, to make changes based on their CGM data that improved glycemic control, potentially obviating the need to escalate therapy.
These modest benefits were not sustained at 6 months, suggesting that additional rtCGM is required for durable glycemic benefits.

\section{DIGITAL FEATURES}

This article is published with digital features, including a summary slide, to facilitate understanding of the article. To view digital features for this article go to https://doi.org/10.6084/ m9.figshare.14619045.

\section{INTRODUCTION}

Current guidelines suggest metformin and comprehensive lifestyle modification as the initial approach to type 2 diabetes (T2D) management [1], with the aim of reaching a target HbA1c of less than $7.0 \%$ to $7.5 \%$ [2]. Since T2D is a progressive disease, therapy adjustments are expected. Management guidelines from the National Institute for Health and Care Excellence (NICE) recommend that drug treatment should be intensified if hemoglobin A1c (HbA1c) levels are not adequately controlled and rise to $7.5 \%$ or higher in adults with T2D [3]. Within 3-4 years of initiating metformin, most patients require a second-line antidiabetic drug [4]. However, data from real-world studies show that intensification of therapy is commonly delayed [5-9] and, even when insulin is introduced, adherence is poor and optimal glycemic control is often not obtained or sustained [6, 10-14].

The role of glucose monitoring to facilitate improved lifestyle choices and prevent therapy intensification is unclear for patients with T2D not using insulin. Numerous studies have shown a lack of benefit of self-monitoring of blood glucose (SMBG) [15-19]. However, a common theme in these studies is that patients and/or clinicians failed to utilize the glucose data. There is evidence that shows this is also a problem in the real world [20]. Conversely, studies in which SMBG data are used by patients 
and clinicians have shown significant HbA1c reduction [21-23]. In these studies, SMBG is performed before and after meals, providing participants immediate feedback about their food choices [21, 22]. While SMBG may be effective if the data are used to modify behavior and treatment, there are numerous barriers to SMBG testing [24]. Many insurers provide limited coverage for test strips for most non-insulin users and, even with coverage, few patients with T2D perform SMBG at the recommended frequency [25].

Real-time continuous glucose monitoring (rtCGM) represents a considerable advance over SMBG-glucose data are obtained without the need to lance the fingertip and are transmitted effortlessly at regular intervals from a subcutaneous sensor to a nearby receiver or smart device, providing users immediate feedback on their lifestyle choices. Currently available systems are more sophisticated and user-friendly than early-generation systems and some do not require blood glucose calibration. While randomized controlled trial (RCT) data suggest that sustained rtCGM use is beneficial for patients with insulin-treated T2D [26], there are limited data evaluating the effects of intermittently used rtCGM in patients with T2D who are not on insulin.

A study conducted by Ehrhardt et al. [27] was the first to investigate standalone rtCGM use in participants with T2D not on insulin. Participants used an early-generation CGM system intermittently for 12 weeks, including four cycles in which CGM was worn for 2 out of 3 weeks (a total of eight week-long CGM sessions across 12 weeks). The data showed significant and sustained improvements in $\mathrm{HbA1c}$ for those using rtCGM compared to SMBG $[27,28]$. In this study, there were no specific management guidelines provided to patients or to their treating clinicians, suggesting that real-time feedback about the glycemic effects of meals and exercise was sufficient to teach lifestyle skills that resulted in better glycemic control for patients with T2D. A major limitation of this study was that the episodic rtCGM use regimen ( 8 out of 12 weeks) utilized in this study is not reimbursable or commonly used in clinical practice. A randomized study by Cox et al. [29] looked at the benefit of a formal, group-oriented, structured education program in conjunction with rtCGM (worn for five sessions) in 30 subjects with early T2D and found that participants using rtCGM successfully modified their diets and lowered their HbA1c on the basis of their CGM data and the structured education. Glycemic benefits were also observed for adults with T2D who used rtCGM intermittently on an individualized schedule and engaged with a diabetes coach via a virtual diabetes clinic; greater benefit was observed for those who used rtCGM for more than 30 days across approximately 5 months $[30,31]$.

Accordingly, there is additional need to ascertain if benefits of episodic rtCGM use in non-insulin-using adults with poorly controlled T2D can be observed outside of a structured coaching program and using a more conventional (and reimbursable) monitoring approach. The COntinuous Glucose Monitoring \& Management In TypE 2 Diabetes (COMMITED; clinicaltrials.gov NCT03620357) study was a pilot study of methods designed to be easily translatable to routine medical practice. Its objective was to determine if limited, episodic use of rt-CGM (10 days a month for 3 months) and experiential learning in patients failing multiple non-insulin medications would improve their lifestyle choices and/or enhance medication adherence and result in glycemic benefit.

\section{METHODS}

\section{Enrollment Criteria}

We enrolled adult patients with T2D who were receiving two or more oral antidiabetic drugs at eight North American endocrinology clinics. Major eligibility criteria included age $30+$ years; diagnosis of T2D; treated with two or more noninsulin antidiabetic drugs; HbA1c $\geq 7.8 \%$ and $\leq 10.5 \%$ by local laboratory or point of care; stable body weight over the past 3 months; English speaking; and owner of a compatible smart device for CGM data display (receivers were not used). Exclusion criteria included use of insulin; prior CGM use (past professional 
CGM use was acceptable); pregnancy; and estimated glomerular filtration rate (eGFR) $<30 \mathrm{~mL} / \mathrm{min} / 1.73 \mathrm{~m}^{2}$. The protocol and Health Insurance Portability and Accountability Act (HIPAA)-compliant informed consent forms were approved by Salus IRB (Board Numbers IRB00006833, IRB00006834, and IRB00009473), who agreed with the determination of non-significant risk for the device study. Written informed consent was obtained from each participant in accordance with the Code of Federal Regulations (CFR) Title per Department of Health and Human Services (DHSS) regulations (45 CFR 46, subpart A \& D). Subjects were asked to sign state-specific forms, such as Subject's Bill of Rights, or equivalent, (if applicable) and HIPAA authorization form, if not included in the site's consent template. Subjects were provided the opportunity to review these documents prior to coming to the clinical site. The investigator or designee explained the purpose and duration of the study, the study procedures and subject requirements, and the potential risks and benefits. The consenting process was documented for each participant and a copy of the consent was provided to the participant. All procedures followed were in accordance with the ethical standards of the institutional review board and ethics committee on human experimentation (institutional and national) and with the Helsinki Declaration of 1964, as revised in 2013.

\section{Study Design}

The study was a prospective, randomized, pilot clinical trial that included a run-in phase, a study phase, and a follow-up visit (Fig. S1 in the supplementary material). At enrollment and run-in, participants completed quality-of-life questionnaires (data not reported here) and wore Dexcom G6 CGM (Dexcom Inc, San Diego, CA) in a blinded, study mode for one 10-day wear session to collect baseline CGM data. Participants were then randomized in a 2:1 ratio to rtCGM or SMBG groups. The rtCGM group used unblinded rtCGM (Dexcom G6) for 10 days each at weeks 0,4 , and 8. Participants were provided learning modules with each
CGM wear period to facilitate experiential learning in response to their glucose data (Fig. S2 in the supplementary material). The SMBG group managed diabetes with daily SMBG and wore blinded rtCGM at week 8 for comparison of CGM metrics to the rtCGM group. Both groups had HbA1c collected at weeks 0 and 12 . Per protocol, medication changes were disallowed for participants in either group unless required for safety. There were phone visits with a study-site clinician at weeks 2, 6 and 10 in both groups to review the SMBG or rtCGM data. During these calls, there were structured discussions about what the subjects learned from their glucose monitoring, what changes were made in response to the data, and what the study clinician observed. After week 12, participants were followed via usual care by their clinician (medication changes were allowed) and returned for a follow-up visit at month 9 to obtain local lab or point-ofcare HbA1c. Reportable adverse events included all device-or study-related adverse events, severe hypoglycemia (defined as an event that required assistance from another person to administer carbohydrates or other resuscitative action), diabetic ketoacidosis or severe hyperglycemia if treatment was received at a healthcare facility, and serious adverse events regardless of causality.

\section{Data Analysis}

Participants with at least 5 days of CGM data within each active wear period were included in the analysis. Participants with less than 5 days of cumulative CGM data (initial and replacement devices) in an active wear period were excluded from the analysis. Independent $t$ tests were performed for comparisons between baseline (week 0) and week $12 \mathrm{HbA} 1 \mathrm{c}$ values, week 0 and month $9 \mathrm{HbA1c}$ values, and the percentage of participants reaching an HbA1c of less than $7.5 \%$ and $7.0 \%$ tested at a one-sided significance level of 0.05 , using SAS ${ }^{\circledR}$ software, version 9.4 (SAS Institute, Inc., Cary, NC). Percentage time in various CGM glucose ranges was calculated as the proportion of sensor glucose values within that range, compared to the total number of 
Table 1 Participant demographics and baseline characteristics

\begin{tabular}{|c|c|c|c|c|}
\hline Characteristics & Statistics & CGM $(n=46)$ & SMBG $(n=24)$ & Overall $(n=70)$ \\
\hline \multirow[t]{4}{*}{ Age } & $n$ & 46 & 24 & 70 \\
\hline & Mean (SD) & $58.9(11.8)$ & $60.9(9.5)$ & $59.5(11.1)$ \\
\hline & Median & 59.5 & 61.1 & 59.7 \\
\hline & Min, $\max$ & $35.5,80.6$ & $45.3,80.3$ & $35.5,80.6$ \\
\hline \multicolumn{5}{|l|}{ Gender } \\
\hline Male & $n(\%)$ & $27(58.7 \%)$ & $10(41.7 \%)$ & $37(52.9 \%)$ \\
\hline Female & $n(\%)$ & $19(41.3 \%)$ & $14(58.3 \%)$ & $33(47.1 \%)$ \\
\hline \multicolumn{5}{|l|}{ Race } \\
\hline Asian & $n(\%)$ & $9(19.6 \%)$ & $1(4.2 \%)$ & $10(14.3 \%)$ \\
\hline Black or African American & $n(\%)$ & $5(10.9 \%)$ & $0(0.0 \%)$ & $5(7.1 \%)$ \\
\hline Native Hawaiian or Other Pacific Islander & $n(\%)$ & $1(2.2 \%)$ & $1(4.2 \%)$ & $2(2.9 \%)$ \\
\hline White & $n(\%)$ & $31(67.4 \%)$ & $21(87.5 \%)$ & $52(74.3 \%)$ \\
\hline Other & $n(\%)$ & $0(0.0 \%)$ & $1(4.2 \%)$ & $1(1.4 \%)$ \\
\hline \multicolumn{5}{|l|}{ Ethnicity } \\
\hline Hispanic or Latino & $n(\%)$ & $12(26.1 \%)$ & $8(33.3 \%)$ & $20(28.6 \%)$ \\
\hline Not Hispanic or Latino & $n(\%)$ & $34(73.9 \%)$ & $16(66.7 \%)$ & $50(71.4 \%)$ \\
\hline \multirow[t]{4}{*}{ BMI $\left(\mathrm{kg} / \mathrm{m}^{2}\right)$} & $n$ & 46 & 24 & 70 \\
\hline & Mean (SD) & $31.6(6.9)$ & $33.1(5.8)$ & $32.1(6.6)$ \\
\hline & Median & 29.9 & 32.8 & 31.0 \\
\hline & Min, $\max$ & $19.6,50.0$ & $24.2,46.2$ & $19.6,50.0$ \\
\hline \multirow[t]{4}{*}{ Diabetes duration (years) } & $n$ & 46 & 24 & 70 \\
\hline & Mean (SD) & $13.9(11.0)$ & $12.3(6.7)$ & $13.4(9.7)$ \\
\hline & Median & 11.0 & 11.0 & 11.0 \\
\hline & Min, $\max$ & $2.0,59.0$ & $2.0,23.0$ & $2.0,59.0$ \\
\hline
\end{tabular}

available sensor glucose values in that sensor wear session. As a result of the small sample size of this pilot study, statistical analysis of between-group comparisons was limited to HbA1c endpoints, and analyses of CGM metrics were only descriptive.

\section{RESULTS}

\section{Participant Demographics}

A total of 70 participants were enrolled ( $n=46$ rtCGM; $n=24$ SMBG) from eight North 
Table 2 HbA1c outcomes-baseline to week 12

\begin{tabular}{|c|c|c|c|c|c|c|c|}
\hline \multirow{2}{*}{$\begin{array}{l}\text { Primary } \\
\text { outcome }\end{array}$} & \multicolumn{3}{|l|}{ CGM } & \multicolumn{3}{|l|}{ SMBG } & \multirow[t]{2}{*}{$p$ value } \\
\hline & $\begin{array}{l}\text { Baseline } \\
(n=44)\end{array}$ & $\begin{array}{l}\text { Week 12 } \\
(n=44)\end{array}$ & $\begin{array}{l}\text { Change from } \\
\text { baseline to week } 12\end{array}$ & $\begin{array}{l}\text { Baseline } \\
(n=23)\end{array}$ & $\begin{array}{l}\text { Week 12 } \\
(n=23)\end{array}$ & $\begin{array}{l}\text { Change from } \\
\text { baseline to week } 12\end{array}$ & \\
\hline $\mathrm{A} 1 \mathrm{C}$ & & & & & & & 0.74 \\
\hline $\begin{array}{r}\text { Mean } \\
(\mathrm{SD})\end{array}$ & $8.4(0.7)$ & $8.0(1.1)$ & $-0.5(0.9)$ & $8.5(0.8)$ & $8.1(1.0)$ & $-0.3(0.7)$ & \\
\hline $\begin{array}{c}\text { Median } \\
(\mathrm{IQR})\end{array}$ & $8.4(0.8)$ & $7.9(1.2)$ & $-0.5(1.3)$ & $8.3(1.2)$ & $8.0(0.8)$ & $-0.2(1.1)$ & \\
\hline
\end{tabular}

1

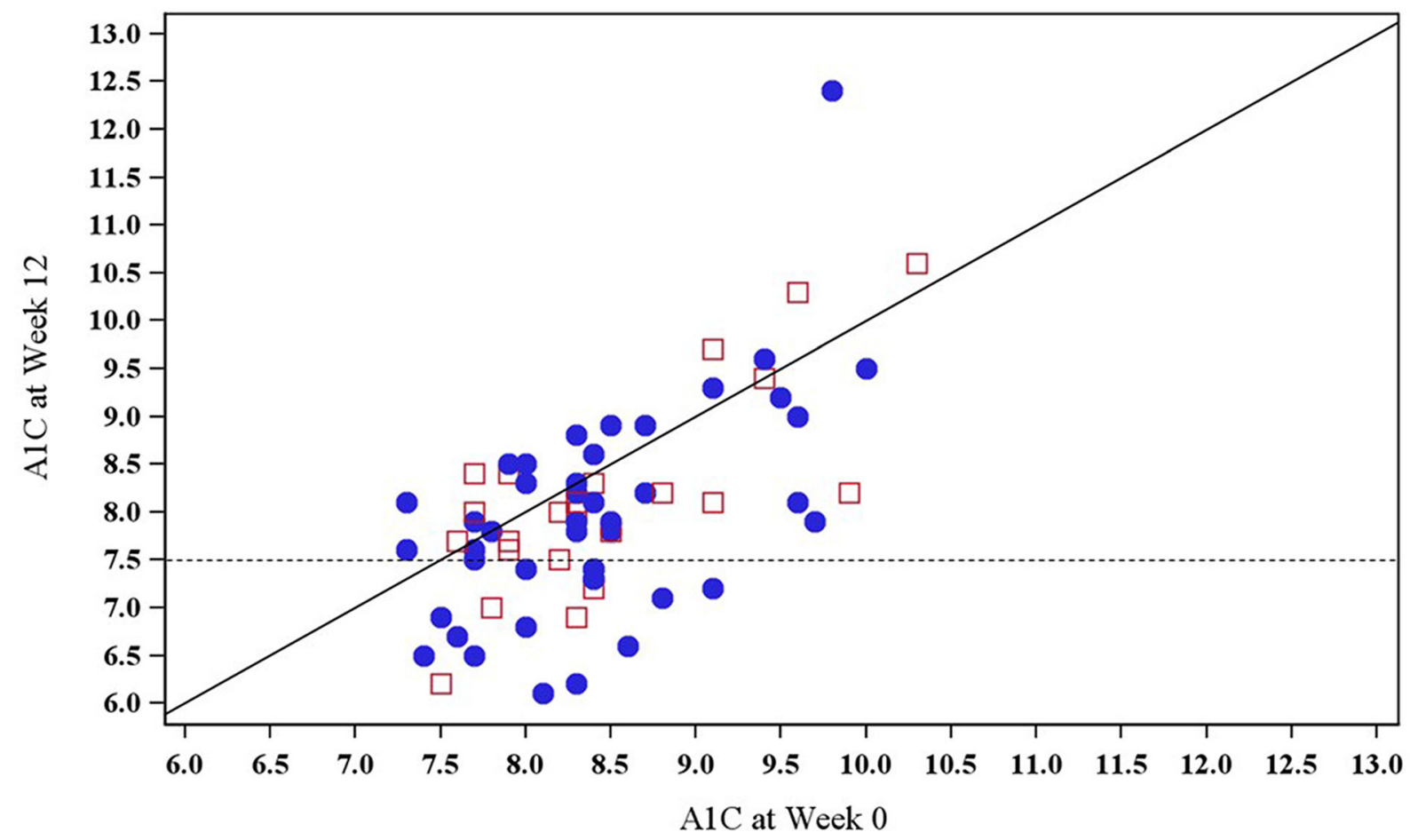

Fig. $1 \mathrm{HbAlc}$ at baseline and week 12; CGM $(n=44)$ and SMBG $(n=23)$. Scatterplot of week 12 hemoglobin Alc (HbAlc) levels by baseline HbAlc level. The horizontal dotted line indicates an HbAlc goal of 7.5\%.

American sites starting in September 2018 and data were available from $68 \quad(n=45$ rtCGM; $n=23$ SMBG). One participant in the rtCGM group did not have at least 5 days of sensor wear
Points below, above, and on the diagonal solid line represent cases in which the week $12 \mathrm{HbAlc}$ level was lower, higher, and the same as the baseline $\mathrm{HbAlc}$ level, respectively

during an active wear period and one participant in the SMBG group had rtCGM initiated by mistake. Baseline characteristics are shown in Table 1. Enrolled participants were using two or 


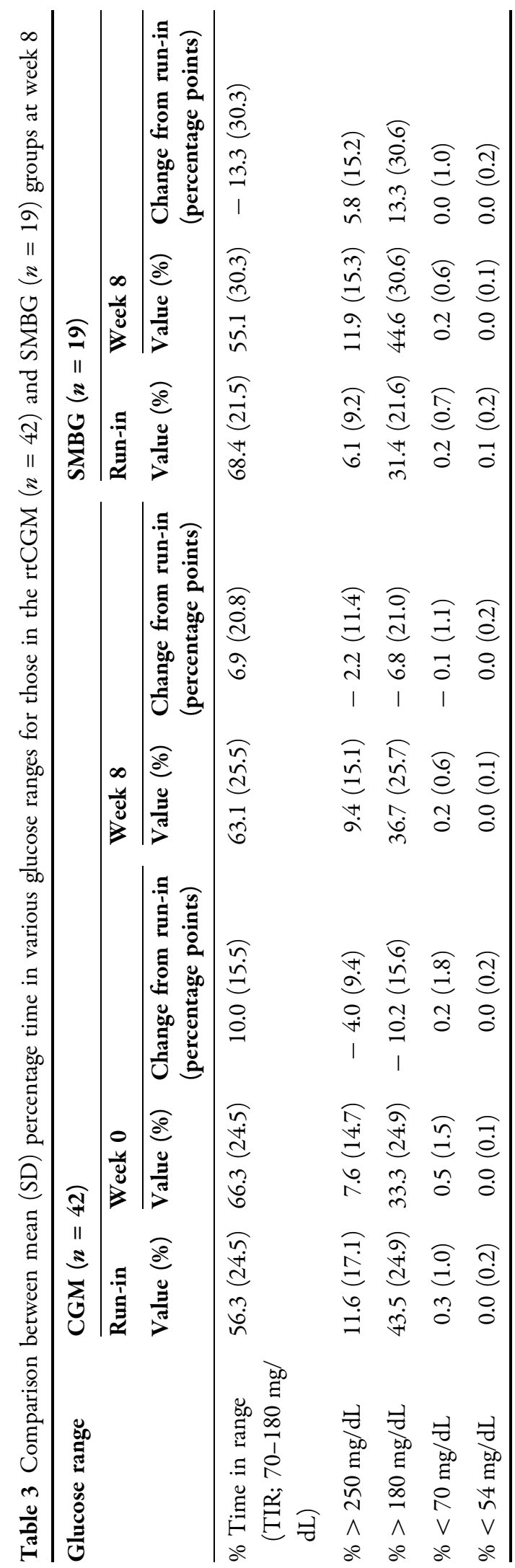

more non-insulin antihyperglycemic medications, most commonly biguanides, sulfonylureas, sodium/glucose cotransporter 2 (SGLT2) inhibitors, and dipeptidyl peptidase 4 (DPP4) inhibitors (Table S1 in the supplementary material).

\section{HbA1c}

Mean (SD) and median (IQR) change in HbA1c from baseline to week 12 was - $0.5(0.9) \%$ and $-0.5(1.3) \%$ for those in the rtCGM group and $-0.3(0.7) \%$ and $-0.2(1.1) \%$ for those in the SMBG group, but the between-group difference was not significant $(p=0.74 ;$ Table 2$)$. At week 12, a greater proportion of those randomized to rtCGM than those managing diabetes with SMBG met HbA1c goals of less than $7 \%(18.2 \%$ vs $8.7 \%$, rtCGM vs SMBG; $p=0.26)$ and less than $7.5 \%$ (34.1\% vs $17.4 \%$, rtCGM vs SMBG; $p=0.12$ ) (Fig. 1 ). HbA1c reductions were not sustained at month 9; mean (SD) and median (IQR) change in HbA1c from baseline to month 9 was $-0.2(0.9) \%$ and $-0.2(0.9) \%$, respectively, for those in the rtCGM group and $+0.1(1.3) \%$ and $+0.1(1.6) \%$, respectively, for those in the SMBG group (between-group $p=0.79$ ).

\section{CGM Metrics and Glucose Monitoring}

CGM data were available for both the rtCGM and SMBG groups prior to randomization (runin) and week 8; 90\% of participants had CGM readings up to day 10 . CGM data collected for the SMBG group were from blinded CGM for both time points, whereas they were from blinded CGM (run-in) and unblinded CGM (week 8) for the rtCGM group. Time spent in hypoglycemia was negligible at run-in, even for those on sulfonylureas or a meglitinide. Participants on sulfonylureas or a meglitinide $(n=44)$ spent a mean (SD; min-max) percentage time below $70 \mathrm{mg} / \mathrm{dL}$ of $0.37 \%$ (1.04\%; 0.0-5.3\%). By contrast, those not on sulfonylureas or a meglitinide $(n=20)$ spent a mean (SD; minmax) percentage time below $70 \mathrm{mg} / \mathrm{dL}$ of $0.05 \%$ $(0.15 \% ; 0.0-0.65 \%)$, precluding making any meaningful conclusions about differences in 
time spent in hypoglycemia between the two groups at week 8 .

The rtCGM group experienced a clinically meaningful, more than 5-percentage-point increase in TIR from run-in to week $8(56.3 \%$ vs $63.1 \%$, run-in vs week 8 ), realized primarily as a reduction in hyperglycemia with a glucose level above $180 \mathrm{mg} / \mathrm{dL}$ (Table 3). By contrast, the SMBG group experienced a considerable decrease in TIR $(68.4 \%$ to $55.1 \%$, run-in vs week 8). Of note, when participants in the rtCGM group transitioned from blinded CGM at run-in to rtCGM at randomization (week 0), there was an immediate 10-percentage-point increase in mean (SD) TIR, from 56.3 (24.5)\% to $66.3(24.5) \%$.

\section{Adverse Events}

No serious adverse events (SAEs) occurred in either group during the active wear period. In the rtCGM group, there were two adverse events (AEs) among two participants-one instance of excessive skin irritation that was device and study related, and one dental extraction that was not related to the study, device, or disease. In the SMBG group, there were six AEs among four participants-one instance of excessive skin irritation from an adhesive overlay that was study related, one hypoglycemic event that was disease related, and one instance each of coronary artery disease, fibromyalgia, poison ivy rash, and low iron level that were not study, device, or disease related.

\section{DISCUSSION}

Episodic CGM is often called professional CGM; data from such systems can be blinded and used predominantly by treating clinicians or displayed in real time to empower the user. This trial assessed the glycemic benefit of episodic rtCGM on adults with T2D treated with multiple oral antihyperglycemic medications and in poor glycemic control with $\mathrm{HbA} 1 \mathrm{c} \geq 7.8 \%$. The study utilized experiential learning-participants were instructed to complete worksheets, record the postprandial increases in glucose levels, and consider factors that contributed to hyperglycemia. The remote communications with the clinicians at clinical sites were modeled after those used in a study of structured SMBG use by non-insulin-treated patients with T2D followed by family practitioners [21]. They encouraged self-learning and self-reflection via prompts such as, "What did you learn from your glucose data and what changes did you make in response to the data?" (Fig. S2 in the supplementary material).

We observed a small, non-statistically significant reduction in median and mean HbA1c after 12 weeks of rtCGM use. Unlike the Vigersky study $[27,28]$, the observed HbA1c reduction diminished after rtCGM was withdrawn. The differences may be related to frequency of use. In the Vigersky study, participants had eight wear sessions while this study only utilized three wear sessions. Despite the modest HbA1c reduction in the current study, a numerically greater proportion of episodic rtCGM users reached HbA1c goals of less than $7.5 \%$ and less than $7.0 \%$ than participants who managed their diabetes with SMBG. Time spent in range increased for participants within the rtCGM group and decreased in the SMBG group. The rapid increase of TIR with the initial rtCGM use has been observed even with the earliest generation CGM devices [32]. These data demonstrate that even non-insulin users with T2D are able to learn from their CGM data and make lifestyle changes to diminish hyperglycemic excursions. When translated to clinical practice, greater benefits of episodic rtCGM use may be observed; the CGM data were not used in this study to adjust therapy as the study was designed to assess lifestyle changes made by the study participants.

The lack of improvement in glycemic control between week 12 and month 9 is informative and highlights clinical inertia. During this freeliving follow-up period, participants were seen by their usual clinicians; diabetes care was routine and medication changes or additions could have occurred. However, only five participants in the SMBG group and eight participants in the rtCGM group initiated additional medications (data not shown), despite the fact that the majority of participants in both groups were not meeting HbA1c goals. 
The study has several strengths. First, the study design is meant to translate to real-world, episodic rtCGM use (professional CGM) and the pattern of use fits with current procedural codes (CPT codes 95250 and 95251, Table S2 in the supplementary material). The learning modules and structured remote visits could readily be provided and performed by primary care physicians or by diabetes specialists. Second, the study had broad inclusion criteria and participants used a wide range of diabetes medications. The study has a number of limitations. The sample size was small and although sufficient for this exploratory study, impacted the ability to draw statistically significant conclusions. The study only assessed user responses to the CGM data-CGM data were not used to adjust therapy. Lastly, a few participants had 12-week visits in early- to mid-2020, after the COVID-19 pandemic commenced. We cannot comment on the effect of the pandemic on changes in HbA1c. Although we expect such impact to be minor, given the small TIR improvements observed among real-world G6 users during the early pandemic [33], the social and economic disruption from the pandemic may have had a profound impact on some participants.

\section{CONCLUSION}

T2D is a progressive disease that requires medication titrations and often therapy intensification. Insulin therapy is typically the last resort and its use is often delayed at the expense of prolonged hyperglycemia. This pilot study demonstrates that while episodic rtCGM use may not result in significant improvements in HbA1c for all patients with poorly controlled $\mathrm{T} 2 \mathrm{D}$, some patients were able to realize HbA1c goals. Thus, episodic rtCGM provides a nonpharmacologic option for people failing multiple non-insulin hypoglycemic agents. Further studies with more participants are needed to determine whether a greater frequency of rtCGM use, episodic use over a long period, enhanced coaching (such as in $[30,31]$ ), or medication adjustment based on the CGM data would provide greater and more durable glycemic benefit, obviating the need for therapy intensification.

\section{ACKNOWLEDGEMENTS}

We thank the participants of the study.

Funding This study was supported by research grants from Dexcom, Inc., who also funded the journal's Rapid Service Fee.

Medical Writing and/or Editorial Assistance We thank Sarah Puhr and John Welsh for their assistance in preparing the manuscript.

Authorship Contributions and Other
Acknowledgements All named authors meet the International Committee of Medical Journal Editors (ICMJE) criteria for authorship for this article, take responsibility for the integrity of the work as a whole, and have given their approval for this version to be published. We thank Wenhao Dong, Heather Tecca, Qinyu Chen, and Xiaohe Zhang for their contributions to the statistical analysis; and we thank Polachai Ernie Sakchalathorn for his assistance in running the study.

Disclosures Qianqian Deng, Stayce Beck, and David Price are employees of Dexcom, Inc. Mark Kipnes has no disclosures.

Compliance with Ethics Guidelines The protocol and Health Insurance Portability and Accountability Act (HIPAA)-compliant informed consent forms were approved by Salus IRB (Board Numbers IRB00006833, IRB00006834, and IRB00009473) who agreed with the determination of non-significant risk for the device study. Written informed consent was obtained from each participant in accordance with the Code of Federal Regulations (CFR) Title per Department of Health and Human Services (DHSS) regulations (45 CFR 46, subpart A \& D). Subjects were asked to sign state-specific forms, such as Subject's Bill of Rights, or equivalent, (if applicable) and HIPAA authorization form, if not included in the site's consent template. Subjects were provided the 
opportunity to review these documents prior to coming to the clinical site. The investigator or designee explained the purpose and duration of the study, the study procedures and subject requirements, and the potential risks and benefits. The consenting process was documented for each participant and a copy of the consent was provided to the participant. All procedures followed were in accordance with the ethical standards of the institutional review board and ethics committee on human experimentation (institutional and national) and with the Helsinki Declaration of 1964, as revised in 2013.

Data Availability The datasets generated during and/or analyzed during the current study are not publicly available because this was a pilot/feasibility study. Further studies from which the data may be available from the corresponding author upon reasonable request are being considered.

Open Access. This article is licensed under a Creative Commons Attribution-NonCommercial 4.0 International License, which permits any non-commercial use, sharing, adaptation, distribution and reproduction in any medium or format, as long as you give appropriate credit to the original author(s) and the source, provide a link to the Creative Commons licence, and indicate if changes were made. The images or other third party material in this article are included in the article's Creative Commons licence, unless indicated otherwise in a credit line to the material. If material is not included in the article's Creative Commons licence and your intended use is not permitted by statutory regulation or exceeds the permitted use, you will need to obtain permission directly from the copyright holder. To view a copy of this licence, visit http://creativecommons.org/licenses/bync/4.0/.

\section{REFERENCES}

1. American Diabetes Association. 9. Pharmacologic approaches to glycemic treatment: standards of medical care in diabetes-2020. Diabetes Care. 2020;43(Suppl 1):S98-110.
2. Desai U, Kirson NY, Kim J, et al. Time to treatment intensification after monotherapy failure and its association with subsequent glycemic control among 93,515 patients with Type 2 diabetes. Diabetes Care. 2018;41(10):2096-104.

3. NICE. NICE Clinical Guideline. Type 2 diabetes in adults: management. London: National Institute for Health and Care Excelence; 2015 (Contract No.: NG28).

4. Montvida O, Shaw J, Atherton JJ, Stringer F, Paul SK. Long-term trends in antidiabetes drug usage in the U.S.: real-world evidence in patients newly diagnosed with type 2 diabetes. Diabetes Care. 2018;41(1):69-78.

5. Kostev K, Golz S, Scholz BM, Kaiser M, Pscherer S. Time to insulin initiation in type 2 diabetes patients in 2010/2011 and 2016/2017 in Germany. J Diabetes Sci Technol. 2019;13(6):1129-34.

6. Khunti K, Damci T, Meneghini L, Pan CY, Yale JF, Group SS. Study of Once Daily Levemir (SOLVE): insights into the timing of insulin initiation in people with poorly controlled type 2 diabetes in routine clinical practice. Diabetes Obes Metab. 2012;14(7):654-61.

7. Khunti K, Millar-Jones D. Clinical inertia to insulin initiation and intensification in the UK: a focused literature review. Prim Care Diabetes. 2017;11(1): 3-12.

8. Mata-Cases M, Franch-Nadal J, Real J, et al. Therapeutic inertia in patients treated with two or more antidiabetics in primary care: factors predicting intensification of treatment. Diabetes Obes Metab. 2018;20(1):103-12.

9. $\mathrm{Fu} \mathrm{AZ,} \mathrm{Sheehan} \mathrm{JJ.} \mathrm{Treatment} \mathrm{intensification} \mathrm{for}$ patients with type 2 diabetes and poor glycaemic control. Diabetes Obes Metab. 2016;18(9):892-8.

10. Cramer JA. A systematic review of adherence with medications for diabetes. Diabetes Care. 2004;27(5): 1218-24.

11. Kostev K, Dippel FW, Rathmann W. Glycemic control after initiating basal insulin therapy in patients with type 2 diabetes: a primary care database analysis. Diabetes Metab Syndr Obes. 2015;8: $45-8$.

12. Pantalone KM, Misra-Hebert AD, Hobbs TM, et al. Intensification patterns and the probability of HbA1c goal attainment in type 2 diabetes mellitus: real-world evidence for the concept of 'intensification inertia'. Diabet Med. 2020;37(7):1114-24.

13. Canivell S, Mata-Cases M, Real J, et al. Glycaemic control after treatment intensification in patients 
with type 2 diabetes uncontrolled on two or more non-insulin antidiabetic drugs in a real-world setting. Diabetes Obes Metab. 2019;21(6):1373-80.

14. Meneghini LF, Mauricio D, Orsi E, et al. The Diabetes Unmet Need with Basal Insulin Evaluation (DUNE) study in type 2 diabetes: achieving HbA1c targets with basal insulin in a real-world setting. Diabetes Obes Metab. 2019;21(6):1429-36.

15. Davidson MB, Castellanos M, Kain D, Duran P. The effect of self monitoring of blood glucose concentrations on glycated hemoglobin levels in diabetic patients not taking insulin: a blinded, randomized trial. Am J Med. 2005;118(4):422-5.

16. Towfigh A, Romanova M, Weinreb JE, et al. Selfmonitoring of blood glucose levels in patients with type 2 diabetes mellitus not taking insulin: a metaanalysis. Am J Manag Care. 2008;14(7):468-75.

17. Kleefstra N, Hortensius J, Logtenberg SJ, et al. Selfmonitoring of blood glucose in tablet-treated type 2 diabetic patients (ZODIAC). Neth J Med. 2010;68(1):311-6.

18. Malanda UL, Bot SD, Nijpels G. Self-monitoring of blood glucose in noninsulin-using type 2 diabetic patients. It is time to face the evidence. Diabetes Care. 2013;36(1):176-8.

19. Young LA, Buse JB, Weaver MA, et al. Glucose selfmonitoring in non-insulin-treated patients with type 2 diabetes in primary care settings: a randomized trial. JAMA Intern Med. 2017;177(7):920-9.

20. Grant RW, Huang ES, Wexler DJ, et al. Patients who self-monitor blood glucose and their unused testing results. Am J Manag Care. 2015;21(2):e119-29.

21. Schwedes U, Siebolds M, Mertes G, Group SS. Mealrelated structured self-monitoring of blood glucose: effect on diabetes control in non-insulin-treated type 2 diabetic patients. Diabetes Care. 2002;25(11):1928-32.

22. Polonsky WH, Fisher L. Self-monitoring of blood glucose in noninsulin-using type 2 diabetic patients: right answer, but wrong question: selfmonitoring of blood glucose can be clinically valuable for noninsulin users. Diabetes Care. 2013;36(1):179-82.

23. Duran A, Martin P, Runkle I, et al. Benefits of selfmonitoring blood glucose in the management of new-onset type 2 diabetes mellitus: the St Carlos Study, a prospective randomized clinic-based interventional study with parallel groups. J Diabetes. 2010;2(3):203-11.

24. Price D, Walker $\mathrm{T}$. The rationale for continuous glucose monitoring-based diabetes treatment decisions and non-adjunctive continuous glucose monitoring use. Eur Endocrinol. 2016;12(1):24-30.

25. Karter AJ, Ferrara A, Darbinian JA, Ackerson LM, Selby JV. Self-monitoring of blood glucose: language and financial barriers in a managed care population with diabetes. Diabetes Care. 2000;23(4):477-83.

26. Beck RW, Riddlesworth TD. Continuous glucose monitoring versus usual care in patients with type 2 diabetes receiving multiple daily insulin injections. Ann Intern Med. 2018;168(7):526-7.

27. Ehrhardt NM, Chellappa M, Walker MS, Fonda SJ, Vigersky RA. The effect of real-time continuous glucose monitoring on glycemic control in patients with type 2 diabetes mellitus. J Diabetes Sci Technol. $2011 ; 5(3): 668-75$.

28. Vigersky RA, Fonda SJ, Chellappa M, Walker MS, Ehrhardt NM. Short- and long-term effects of realtime continuous glucose monitoring in patients with type 2 diabetes. Diabetes Care. 2012;35(1): 32-8.

29. Cox DJ, Banton T, Moncrief M, Conaway M, Diamond A, McCall AL. Minimizing glucose excursions (GEM) with continuous glucose monitoring in type 2 diabetes: a randomized clinical trial. J Endocr Soc. 2020;4(11):118.

30 Dixon RF, Zisser H, Layne JE, et al. A virtual type 2 diabetes clinic using continuous glucose monitoring and endocrinology visits. J Diabetes Sci Technol. 2019. https://doi.org/10.1177/1932296819888662.

31. Bergenstal RM, Layne JE, Zisser $\mathrm{H}$, et al. Remote application and use of real-time continuous glucose monitoring by adults with type 2 diabetes in a virtual diabetes clinic. Diabetes Technol Ther. 2021;23(4):1-5.

32. Garg S, Zisser H, Schwartz S, et al. Improvement in glycemic excursions with a transcutaneous, realtime continuous glucose sensor: a randomized controlled trial. Diabetes Care. 2006;29(1):44-50.

33. van der Linden J, Welsh JB, Hirsch IB, Garg SK. Realtime CGM (rtCGM) during the COVID-19 pandemic and its impact on time in range. Diabetes Technol Ther. 2021;23(S1):S1-S7. 